\title{
Simulating the signal of the AMIGA underground muon detector of the Pierre Auger Observatory
}

\author{
A. M. Botti, ${ }^{a, b, *}$ F. Sánchez, ${ }^{a}$ M. Roth, ${ }^{c}$ and A. Etchegoyen ${ }^{a}$ \\ ${ }^{a}$ Instituto de Tecnologías en Detección y Astropartículas (CNEA, CONICET, UNSAM), Buenos Aires, \\ Argentina \\ ${ }^{b}$ Department of Physics, FCEyN, University of Buenos Aires and IFIBA, CONICET, Buenos Aires, \\ Argentina \\ ${ }^{c}$ Karlsruhe Institute of Technology (KIT), Institute for Astroparticle Physics, Karlsruhe, Germany \\ E-mail: ana.botti@iteda.cnea.gov.ar
}

\begin{abstract}
We present a detailed description of the simulation development and validation of the underground muon detector signal for the Pierre Auger Observatory. To this aim, the detection system was thoroughly characterized in the laboratory. It consists of plastic-scintillator strips with optical fibers that conduct light towards silicon photomultipliers whose output is then processed with two complementary read-out channels. These measurements allowed us to design a fast and reliable simulation chain that fully reproduces the signal of single muons impinging on the scintillators.
\end{abstract}

$37^{\text {th }}$ International Cosmic Ray Conference (ICRC 2021)

July 12 th - 23rd, 2021

Online - Berlin, Germany

\footnotetext{
${ }^{*}$ Presenter
} 


\section{Introduction}

The underground muon detector (UMD) is a part of the lower-energy enhancement of the surface detector at the Pierre Auger Observatory [1], named originally as the AMIGA (Auger Muon and Infill for the Ground Array) system [2]. Currently, the infilled grids of water-Cherenkov detectors are referred to as the SD-750 and SD-433 within the AugerPrime upgrade program and the buried scintillators as the UMD. This latter is aimed at obtaining direct measurements of the muonic component in air showers in the energy region of the cosmic-ray spectrum between the "second knee" and the "ankle". The detector layout consists of two triangular grids of paired water-Cherenkov detectors and underground plastic scintillators with spacings of 433 and $750 \mathrm{~m}$. At each position of this underground muon detector three $10^{2} \mathrm{~m}$ modules are buried with $2.3 \mathrm{~m}$ of overburden, each of which consists of 64 plastic scintillator strips of $(400 \times 1 \times 4) \mathrm{cm}^{3}$ with wavelength-shifting optical-fiber coupled to an array of 64 silicon photomultipliers (SiPMs).

During the engineering array phase, the UMD optoelectronics consisted of a 64-pixel multianode PMT with inverter amplifiers and discriminators [3]. For the production phase [4], the detector was updated to use SiPMs and a new set of readout electronics with two acquisition modes: the binary [5] and $A D C$ [6]. In the binary mode, we handle independently the 64 SiPM signals through a pre-amplifier, fast-shaper, and a discriminator, built within each channel of two 32-channel Application-Specific Integrated Circuits (ASICs). The discriminator signal is sampled at $320 \mathrm{MHz}$ (3.125 ns sample time) with a Field-Programmable Gate Array (FPGA) into 64 2048-bits traces. At each sample, a "1"-bit is output if the signal is above the discriminator threshold, set at 2.5 photo-equivalents (PEs) to reject most of the SiPM dark rate [5], and a "0"-bit otherwise. In this working mode, muons are identified as sequences of "1"s in the binary traces.

In the ADC mode, the $64 \mathrm{SiPM}$ analog signals are summed and amplified with low- and high-gain amplifiers. The signals are then sampled at $160 \mathrm{MHz}(6.25 \mathrm{~ns}$ sample time) with two Analog-to-Digital Converters (ADCs) into two waveforms of 1024 samples. The number of muons is then estimated by dividing the signal charge by the mean charge of a single muon.

The simulation software in the Auger official framework for simulation and data analysis ( $\overline{\mathrm{Off}}$ line $)$ [8] has recently been updated to match the final design of the UMD by including the simulation of the SiPMs with the two acquisition modes [9]. In this work, we present the UMD simulation, and we thoroughly describe its validation with laboratory measurements.

\section{The simulation of the underground muon detector}

Each UMD component, represented in the left panel of Fig. 1, is simulated independently using phenomenological or electronic models tuned to laboratory data, as we illustrate in the middle (simulation scheme) and right (data summary) panels of Fig. 1. We simulate the SiPM with a PE generator based on an electronic model [10] fitted to analog data. The optical fiber and scintillator are simulated using a muon generator based on a double-exponential decay law [11] that describes the number of detected PEs, also fitted to analog data. We then include the electronics using transfer functions $[6,12]$ based on the circuit layouts tuned to digital data.

To develop and validate the simulation, we used both digital and analog data acquired in the laboratory. In general, the laboratory setup consisted of one scintillator strip with optical fiber coupled to a SiPM as in the UMD [3]. Two readout electronics were used to extract signals: a 


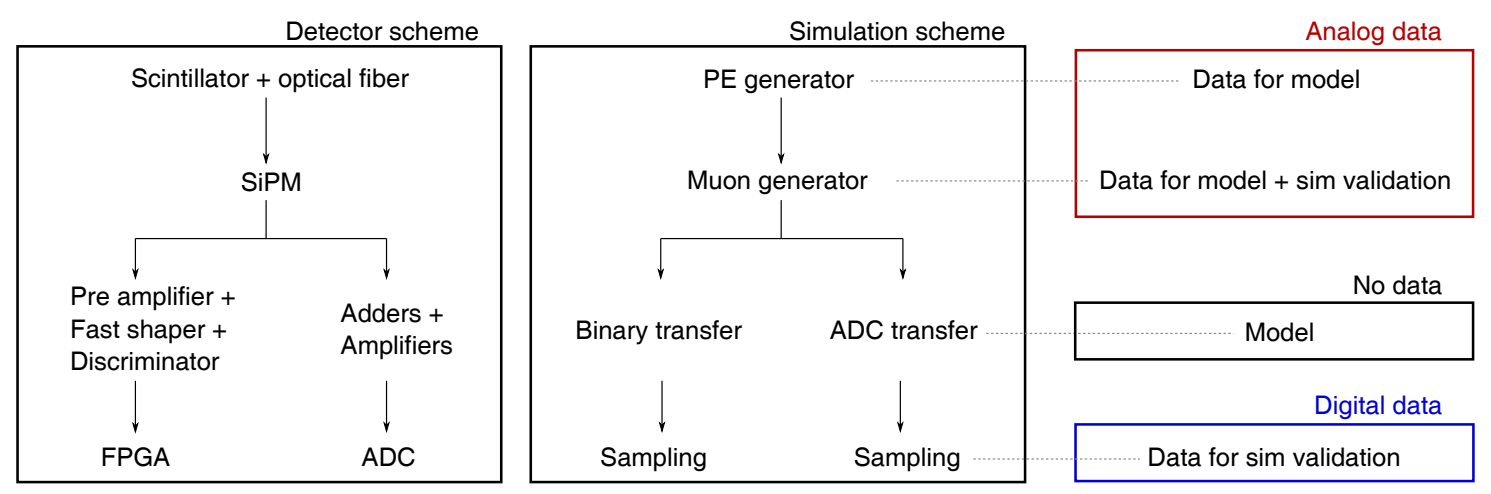

Figure 1: (Left) schematics of the detector components. (Middle) scheme of the UMD simulation steps. (Right) summary of the data used to develop and validate the simulation.

customized inverting amplifier with an oscilloscope to obtain analog data and the standard UMD electronics to acquire digital traces. We covered the scintillator strip with an aluminum container to shield it from environmental light, and we deployed the SiPM and electronics inside a PVC vessel for the same purpose. Due to the optical-fiber attenuation, the number of detected PEs depends on the position on the strip where the impinging muon deposited energy. We used a muon telescope built with two scintillator segments of $(4 \times 4 \times 1) \mathrm{cm}^{3}$, and customized electronics to obtain single-muon data. The telescope generates a trigger signal when a coincidence in the telescope (signal in both segments) is produced, allowing us to select events at specific positions on the strip within a $4 \mathrm{~cm}$ resolution.

The PE generator is based on an electrical model with one rise time and three decay times [10] whose parameters are estimated by fitting the measured dark-rate pulses. The first fall time is a fast decay produced by the coupling of parasitic capacitors to the entrance impedance of the oscilloscope. The second fall time corresponds to the quenching, and the third and slow decay corresponds to the recovery time of the SiPM. We then inputted the mean and standard deviation of these parameters in a random generator to simulate single-PE pulses.

The muon generator is based on a phenomenological model: when a particle impinges on the scintillator, photons are produced with a characteristic decay time of $3.7 \mathrm{~ns}$, and they are absorbed and re-emitted by the optical fiber, which has a characteristic decay time of $3.5 \mathrm{~ns}$. These photons are then propagated through the optical fiber to the SiPM, which detects an average of about $40 \%$ of the arriving photons. To simulate muons, we use a parametrization of the mean number of detected PEs as a function of the position on the scintillator strip where the particle arrives. We then input this mean in a random generator to introduce Poissonian fluctuations. Each detected photon produces a single-PE signal, whose start time is determined by convoluting the decay probability of the scintillator and optical fiber. The resulting muon output is the result of adding all these PE signals.

In the binary acquisition mode, the SiPM output is first processed with a pre-amplifier followed by a fast-shaper and a discriminator. We started simulating the pre-amplifier as $10 \mathrm{MHz}$ active low-pass filter with an amplification factor of $10 \times$. The fast-shaper is simulated as a practical differentiator with a characteristic time of $15 \mathrm{~ns}$ and a maximum gain of about $18 \times$ [12]. We simulate the last steps of the binary electronics (the discriminator and the FPGA) by generating a $3.3 \mathrm{~V}$ signal if the fast-shaper is above the discriminator threshold during more than $1.51 \mathrm{~ns}$ [9] and 

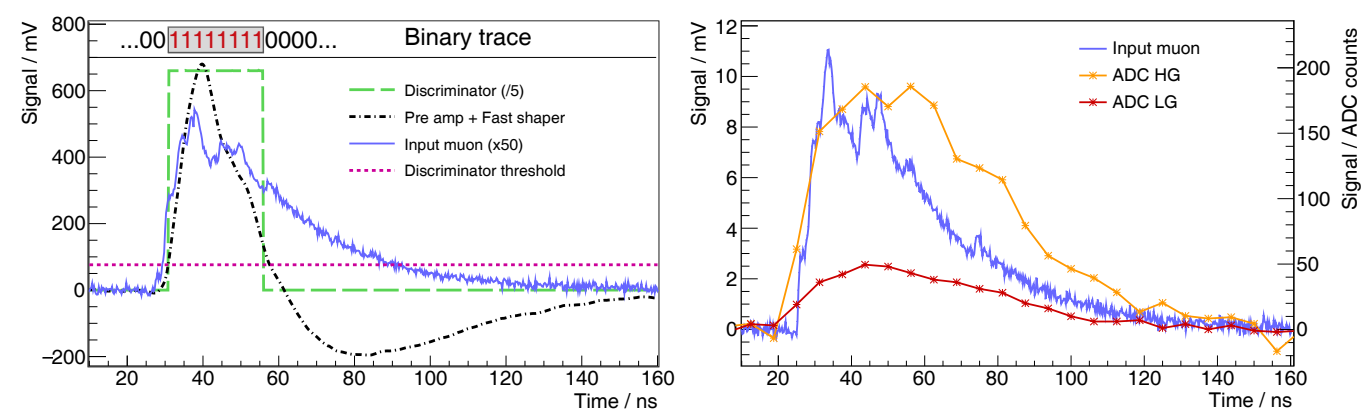

Figure 2: Example of simulated binary (left) and ADC (right) trace at $2 \mathrm{~m}$ on the scintillator strip.

a 0 V signal otherwise. Finally, we output a "1"("0") in the binary trace if the resulting signal is $3.3(0) \mathrm{V}$ with a sampling time of $3.125 \mathrm{~ns}$. The transfer function parameters were then tuned to obtain the best representation of the laboratory data. We present in the left panel of Fig. 2 an example of a simulated single-muon produced at $2 \mathrm{~m}$ from the SiPM with the binary channel. We display the SiPM signal (blue-solid line), the output after the pre-amplifier and the fast-shaper (dotted and dashed black line), the discriminator threshold (dotted-pink line), and its response (dashed-green line), and the FPGA sampling (binary trace).

As we explained in section 1, with the ADC mode we sum in two steps of adders all the analog signals. The first step consists of four adders, each of which processes 16 SiPM outputs, while the second step sums these four outputs into one. Then, we amplify the result with a low- and high-gain amplifier, and we sample the outputs with ADCs into two traces. The adders in the first step introduce a frequency cutoff of about $10 \mathrm{MHz}$, whereas the other steps introduce a frequency cutoff of about $100 \mathrm{MHz}$. In this sense, the electronics response after the first step of adders is rather flat in frequency. Therefore, the whole electronics chain is simulated starting with the transfer function of a low-pass filter with a frequency cut of $12 \mathrm{MHz}$ and a gain of $-2.5(-8.47) \mathrm{dB}[6]$ for the low(high)-gain output, and we tuned the parameters with laboratory data. To simulate the sampling, we group the amplifier outputs in bins of $6.25 \mathrm{~ns}$, and extract the maximum amplitude reached in each sample. We then obtain the ADC counts per sample by diving the signal amplitude by the ADC step of about $0.12 \mathrm{mV} / \mathrm{bit}$. We show in the right panel of Fig. 2 an example of a muon signal at $2 \mathrm{~m}$ from the SiPM. The muon analog signal (blue line) and the output after the ADC are displayed for the low- (red stars) and high-gain (orange stars) amplifiers.

\section{Analog data}

The first step towards the development of the UMD simulation was to obtain a data set of 2000 dark counts. To reject events that do not exclusively contain single-PEs, we removed all events with amplitude and charge above three standard deviations from the single-PE means. The SiPM electrical model was fitted to this data to obtain the PE generator. We illustrate the accuracy of the PE generator in Fig. 3, where the average single-PE measured signal (black squares) and the average over 2000 simulated single-PEs (red circles), along with the difference between the two waveforms (black crosses) is presented. In the inset, we show an overlap of the 2000 simulated PEs used in this figure. The simulation differs between $-5 \%$ and $10 \%$ of the mean measured signal, having the maximum differences when the signal changes abruptly. Still, since this deviation oscillates around 0 , the difference in the integrated signal charge is less than $1 \%$ and less than $3 \%$ when looking at 


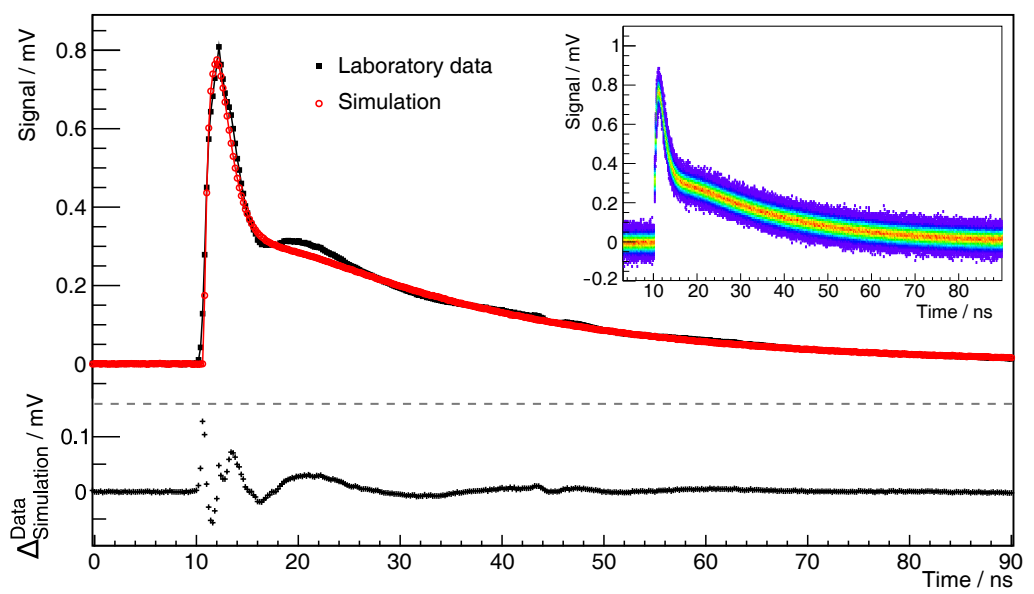

Figure 3: Mean single-PE signal for laboratory data and simulation. In the inset, we show the 2000 simulated single-PE pulses used to calculate the mean. At the bottom, we show the total difference between simulation and data.
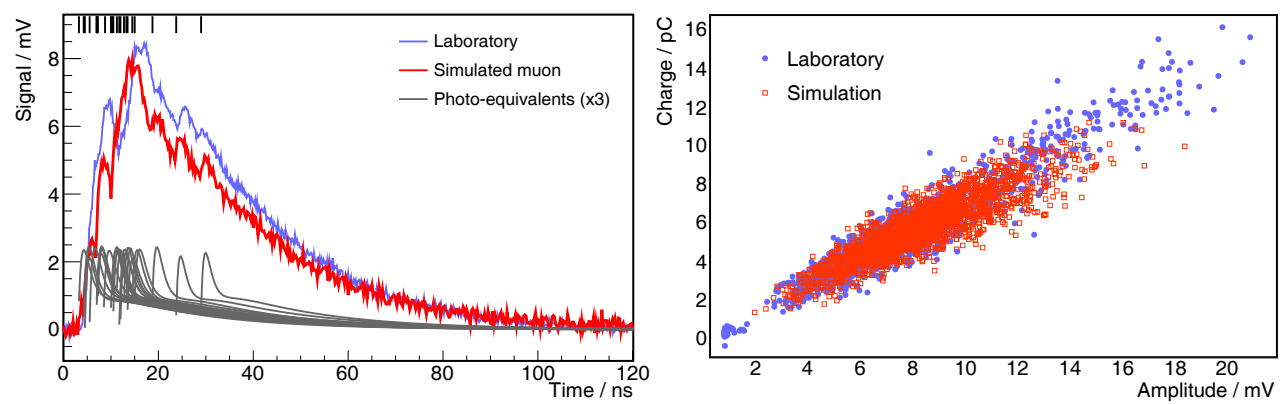

Figure 4: (Left) example muon signal measured and simulated a $2 \mathrm{~m}$ on the scintillator strip. For the simulated signal, we display each PE signal and its arrival time (top x-axis). (Right) correlation between the muon signal charge and amplitude for 2000 events of both laboratory data and simulation.

the maximum amplitude, being these the most relevant parameters for the ADC and binary channel respectively.

As explained in section 2, we use the muon telescope to trigger the system with single muons at different positions on the scintillator strip. An example of a signal measured (blue line) and simulated (red line) at $2 \mathrm{~m}$ from the scintillator edge is presented in the left panel of Fig. 4. For the simulated signal, the single-PE (gray line) and their arrival time (top x-axis) is also displayed: the muon signal structure is determined by the time profile of the arriving photons. In the right panel of Fig. 4, we show the correlation between the charge and amplitude of 2000 muon signals measured (blue circles) and simulated (red squares) at $2 \mathrm{~m}$ on the scintillator strip. The linearity between these two features is apparent as well as the as the agreement between simulation and data around the means. Outliers in laboratory data for high and low amplitudes are not reproduced in the simulation; these represent about $7 \%$ of the data, and, as it will be presented in Section 4, does not have an impact on the accuracy of the whole simulation chain.

The simulation is mainly expected to describe accurately the signal parameters relevant to the estimation of muon densities in air showers using the UMD. Since in the binary mode we implement an amplitude threshold, one of the most important features in the signals is their amplitude. In Fig. 5 top-left panel, we show the mean and standard deviation over 2000 muons of the signal amplitude 

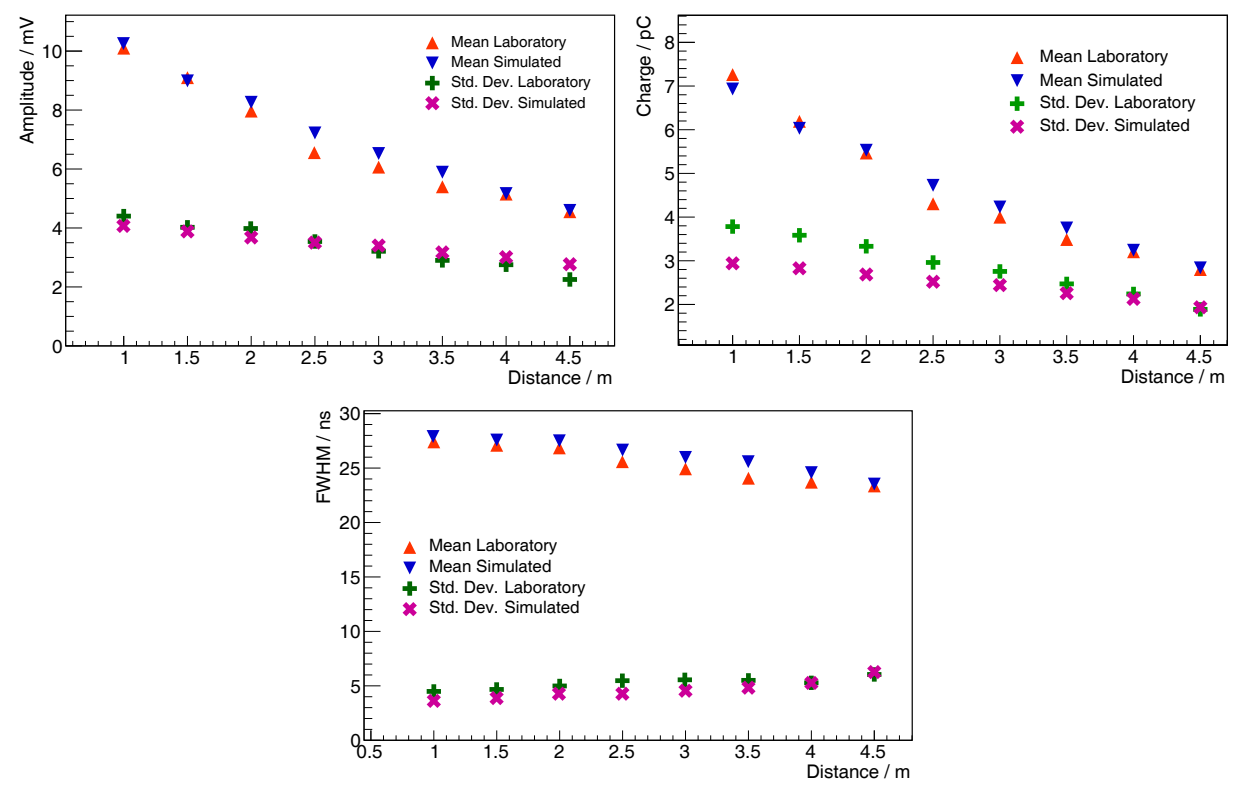

Figure 5: Main features of the muons signals at different positions on the scintillator strip. We show the means and standard deviation for laboratory data and simulations. We display on the top-left panel the muon signal charge, in the top-right panel the muon signal amplitude, and in the bottom, the muon signal full width at half maximum. It is apparent that in all cases there is good agreement between data and simulations.

at different positions on the scintillator strip, using both laboratory data (orange up triangles and green cosses) and simulations (blue down triangles and pink X crosses). On the other hand, the muon signal charge has an impact on the reconstruction of the ADC mode since the number of muons is estimated as the ratio of the total signal charge and the mean charge of the single-muon. In the top-right panel of Fig. 5, we display the mean and standard deviation of the signal charge at different positions on the scintillator strip for both laboratory data and simulations. Finally, the third feature we need to consider is the signal time width, which affects the signal timing of both modes. In the bottom panel of Fig. 5 we show at different positions on the scintillator, the mean and standard deviation of the muon signal full width at half maximum (FWHM) using both laboratory data and simulations. It is apparent from Fig. 5 that there is an agreement between the laboratory data and the simulations, which denotes that the muon simulations provide a good description of the main features of the muon signals.Differences in the charge fluctuations close to the SiPM are then compensated when introducing the fluctuations in the ADC baseline. However, the muon analog signals are only the input of the readout electronics. In the following section, we present the behavior of the UMD simulations after the electronic processing.

\section{Digital data}

The main features relevant to the UMD performance are: the signal width and the muon detection efficiency for the binary channel and the signal charge and saturation for the ADC. To tune and assess the accuracy of the simulated traces after the electronics we acquired digital data using the standard UMD electronics. At each position on the scintillator strip, we obtained 2000 events containing both binary and ADC traces.

In the left panel of Fig. 6, we show the signal width at different positions on the scintillator strip, both with laboratory (full markers) and simulated data (empty markers) and discriminator thresholds 

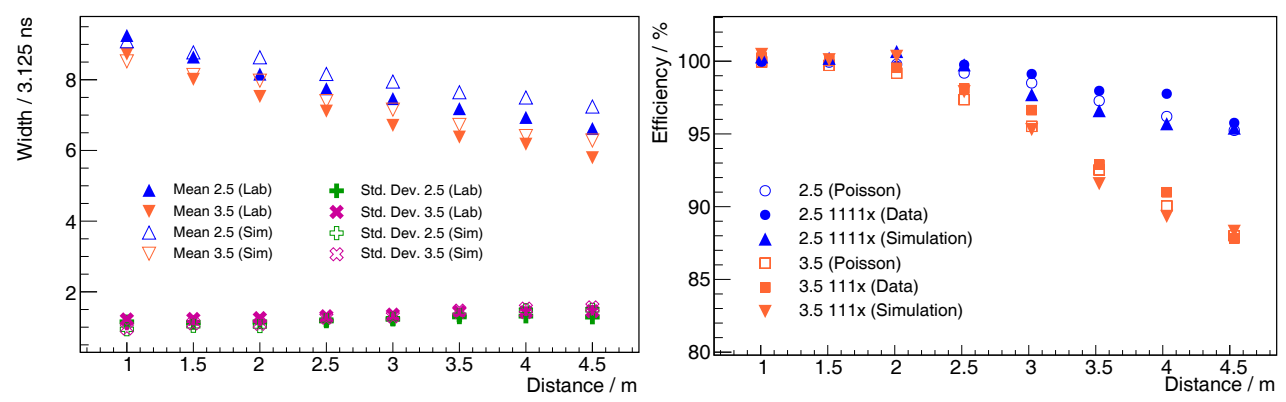

Figure 6: (Left) mean signal width and standard deviation in the binary mode as a function of the position on the scintillator strip with both laboratory and simulated data of single muons. (Right) Efficiency as a function of the position on the scintillator strip estimated with laboratory data, simulations, and a Poissonian prediction.

of 2.5 (blue and green markers) and 3.5 PE (orange and pink markers) ${ }^{1}$. We present the mean value (triangles) and standard deviation (crosses) of the width distribution of single-muon signals. In the right panel, we display the detection efficiency for discriminator thresholds of 2.5 (blue markers) and 3.5 PE (orange markers) using a reconstruction based on the signal width for single muons [9]. The efficiency was estimated using laboratory data (full circles and squares), simulations (triangles), and a Poissonian prediction (empty circles and squares) based on the measured number of PEs as a function of the position on the strip. This prediction consists of integrating a Poissonian distribution between the discriminator threshold (2.5 or 3.5 PE) and infinity. The Poissonian prediction shows that the efficiency loss is mainly produced by muon signals with less than $2.5 \mathrm{PE}$ (or 3.5 PE); the agreement between data and prediction denotes that there is not a significant efficiency loss in the signal processing or the reconstruction analysis. It is apparent that the binary mode simulation provides an accurate description of the binary mode signal width and detection efficiency.

To estimate the number of muons with the ADC channel, we compute the signal charge and divided it by the mean charge of a single-muon. For this reason, the main feature in the ADC mode relevant to the UMD performance is the signal charge and its fluctuations. In the left panel of Fig. 7 we show the mean signal charge of single-muons (circles and squares) and the standard deviation (crosses) as a function of the position on the scintillator strip for laboratory data (full markers) and simulation (empty markers). Data and simulations agree for both high- (orange and green markers) and low-gain (red and blue markers) channels. In addition, we assessed the ADC saturation, which corresponds to the upper limit of the UMD detection range in the right panel of Fig. 7: the signal charge as a function of the number of muons injected simultaneously is displayed for the low- (blue circles) and high-gain (orange squares) channels. The position on the scintillator strip where the muon is injected was selected with a uniform random generator which provides values between $1 \mathrm{~m}$, and $4 \mathrm{~m}$. In dashed-gray lines we show the theoretical linearity between charge and number of injected particles; we identified the saturation point where the signal charge starts to lose linearity to the number of injected muons. The high-gain channel starts to saturate at about 80 simultanous muons, while the low-gain channel starts to saturate at about 300 muons, losing $5 \%$ of the signal with respect to the identity at about 360 muons. It is worth mentioning that

\footnotetext{
${ }^{1}$ The threshold level in the UMD for air shower acquisition is $2.5 \mathrm{PE}$. We used a $3.5 \mathrm{PE}$ threshold only to test the simulation performance
} 

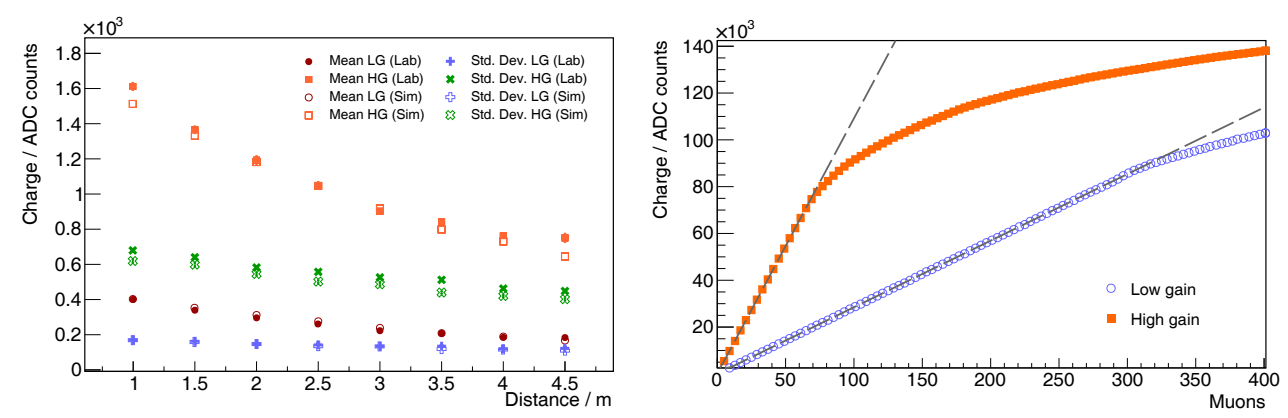

Figure 7: (Left) mean signal charge and standard deviation in the ADC channel as a function of the position on the scintillator strip. (Right) signal charge of simulated data as a function of the number of simultaneously injected muons.

these results are consistent with previous laboratory studies [6] and may be different in the field. Muons in air showers do not arrive concurrently at the detector but with a time profile that may allow the detection of a higher number of particles. On the other hand, muons produce knock-on electrons in the soil, which may increase the total energy deposit per muon, and in turn, produce higher-amplitude signals saturating the detector with a lower number of impinging particles.

\section{Summary}

In this work, we presented the UMD simulation and its validation using laboratory data. We use a single-PE generator which provides the SiPM analog output for single photons. Then, we use a double-exponential decay law and Poissonian fluctuations to generate the number of PEs detected with the SiPM when a muon impinges a scintillator strip. Using the scintillator and optical-fiber characteristic times, single muon signals were simulated and validated using analog laboratory data, showing a good agreement between the two.

After achieving the simulation of the SiPM output signal, we included the electronics response. The transfer functions of both binary and ADC modes were applied to the simulated analog signals to obtain the digital outputs. Data acquired with the standard UMD electronics was used to validate these simulations. We have proved that simulation and laboratory data are compatible. As in the laboratory, the UMD has a detection efficiency of about $98.5 \%$ for single muons and can measure up to about 360 simultaneous muons per module.

\section{References}

[1] The Pierre Auger Collaboration. NIMA 798 (2015) 172-213.

[2] A. Etchegoyen for the Pierre Auger Collaboration. 5 (ICRC2007) 1191-1194.

[3] The Pierre Auger Collaboration. JINST 11 (2016) P02012.

[4] A. M. Botti et al. [for the Pierre Auger Coll.], these proceedings PoS(ICRC2021)233.

[5] The Pierre Auger Collaboration. JINST 12 (2017) P03002.

[6] The Pierre Auger Collaboration. JINST 11 (2020) P01026.

[7] The Pierre Auger Collaboration. JINST 04 (2021) P04003.

[8] S. Argirò, et al. NIM A 580 (2007) 1485-1496.

[9] A. M. Botti, et al. Accepted for publication in JINST (2021) arXiv:2104.13253.

[10] D. Marano and A.D. Grasso. ICECS (2016) 305-308

[11] F. Sánchez and G. Medina-Tanco. NIMA 620 (2010) 182 - 191.

[12] D. Marano et al. Int. J. Circuits Syst. Signal Process 8 (2014) 276-285. 